\title{
Satellite observations of lightning-induced hard X-ray flux enhancements in the conjugate region
}

\author{
R. Bučík ${ }^{1}$, K. Kudela ${ }^{1}$, and S. N. Kuznetsov ${ }^{2}$ \\ ${ }^{1}$ Institute of Experimental Physics, Slovak Academy of Sciences, Watsonova 47, 04001 Košice, Slovakia \\ ${ }^{2}$ Institute of Nuclear Physics, Moscow State University, Vorob'evy Gory, 119899 Moscow, Russia
}

Received: 14 February 2006 - Revised: 10 May 2006 - Accepted: 24 May 2006 - Published: 9 August 2006

\begin{abstract}
Preliminary examination of October-December 2002 SONG (SOlar Neutron and Gamma rays) data aboard the Russian CORONAS-F (Complex Orbital Near-Earth Observations of the Activity of the Sun) low-altitude satellite has revealed many X-ray enhanced emissions $(30-500 \mathrm{keV})$ in the slot region $(L \sim 2-3)$ between the Earth's radiation belts. In one case, CORONAS-F data were analyzed when the intense hard X-ray emissions were seen westward of the South Atlantic Anomaly in a rather wide $L$ shell range from 1.7 to 2.6. Enhanced fluxes observed on day 316 (12 November) were most likely associated with a Major Severe Weather Outbreak in Eastern USA, producing extensive lightning flashes, as was documented by simultaneous optical observations from space. We propose that whistler mode signals from these lightning discharges cause precipitation of energetic electrons from terrestrial trapped radiation belts, which, in turn, produce atmospheric X-rays in the Southern Hemisphere.
\end{abstract}

Keywords. Magnetospheric physics (Energetic particles, precipitating; Magnetosphere-ionosphere interactions)

\section{Introduction}

Hard X-rays from the bremsstrahlung emissions of $\sim \mathrm{MeV}$ electrons precipitating into the atmosphere have been observed both over the auroral zones (Smith et al., 1995; Foat et al., 1998; Millan et al., 2002) during low geomagnetic activity and in the South Atlantic Anomaly (Martin et al., 1974) during a magnetic disturbance. Scattering by the substormexcited electromagnetic ion cyclotron waves has been suggested as a mechanism for the auroral electron precipitation (e.g. Lorentzen et al., 2000).

Correspondence to: R. Bučík

(rbucik@upjs.sk)
Observations of hard X-rays associated with electron precipitation due to lightning flashes are rare. A one-toone correspondence between balloon X-ray $(>30 \mathrm{keV})$ data and ground VLF emissions, triggered by whistlers from lightning, was for the first time, presented by Rosenberg et al. (1971) from an experiment conducted at Siple Station, Antarctica $(L \sim 4.1)$. In a rocket experiment made at Wallops Island, Virginia $(L \sim 2.6)$, Goldberg et al. (1987) observed (with X-ray detectors) electron bursts $(>80 \mathrm{keV})$ that were coincident with lightning detected by nearby ground stations. Geographic intensity maps of hard X-rays (up to $750 \mathrm{keV}$ ) from a low-Earth orbit over mid-latitudes within $\pm 43.3^{\circ}$ and their possible origin in lightning, due to electron precipitation and/or acceleration, has been discussed by Feldman et al. (1996).

There is extensive experimental evidence on the association of individual lightning events with the precipitation of energetic electrons (e.g. Voss et al., 1998, and references therein). The electromagnetic energy originating in lightning discharges escapes into the magnetosphere and propagates as a whistler mode wave, and pitch angle scatters (and thus precipitates) energetic electrons, thereby generating bremsstrahlung hard X-rays.

In the present paper we report on new satellite observations of hard X-rays from the CORONAS-F experiment, which, by association with simultaneous optical observations made on TRMM, may be related to lightning in the geomagnetically conjugate region.

\section{Instrumentation}

The CORONAS-F satellite was launched on 31 July 2001 into a Sun-synchronous polar orbit (inclination $\sim 83^{\circ}$ ) with a period of $\sim 95 \mathrm{~min}$ at $500 \mathrm{~km}$ nominal altitude. The SONG experiment on CORONAS-F contained a large area $\mathrm{CsI}(\mathrm{Tl})$ scintillator $(20 \mathrm{~cm}$ diameter $\times 10 \mathrm{~cm}$ length $)$ for the

Published by Copernicus GmbH on behalf of the European Geosciences Union. 


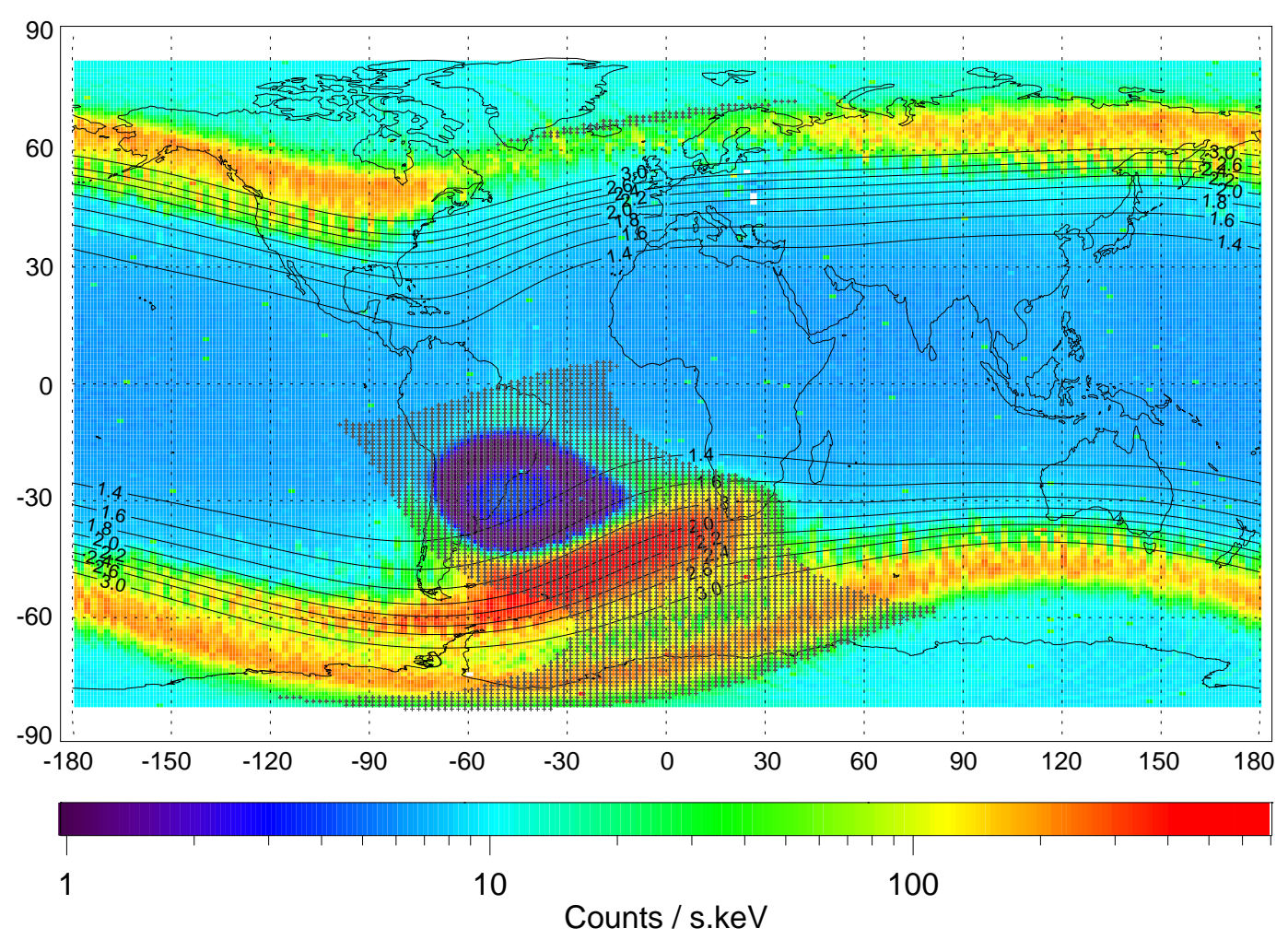

Fig. 1. The colored geographical map of average counts for $60-150 \mathrm{keV}$ X-rays measured by CORONAS-F throughout October-December, 2002 in the altitude range of $440-490 \mathrm{~km}$. Note in the South Atlantic Anomaly the SONG instrument was saturated by high fluxes of energetic $(>50 \mathrm{MeV})$ protons (purple and blue). The footprints of several $L$ shells between 1.4 and 3.0 and the region of stable trapping (crosshatched) at $500 \mathrm{~km}$ are shown for reference. The location of the stable trapping area at an altitude of $500 \mathrm{~km}$ is adopted from Bučík et al. (2005). It consists of set of $L, B$ values (determined by IGRF 1990), for which the minimum longitude traced altitude is above $100 \mathrm{~km}$. Small white strips in the Eastern Europe mean no data available for these regions, due to operative controls when the satellite passed over Moscow. Note that the adjacent region is characterized by a reduction in the amount of data. There are less than 10 records per bin in the area clustered at longitudes $20^{\circ}-30^{\circ}$ and latitudes $40^{\circ}-60^{\circ}$.

measurement of energetic neutral radiation from the Sun (Kuznetsov et al., 2002, 2004). The crystal scintillator was viewed by three photomultipliers and was entirely surrounded by a $2-\mathrm{cm}$ thick plastic anticoincidence shield against charged particles. It was viewed by three other photomultiplier tubes.

During calibration the efficiency of the active anticoincidence shield was tested by using atmospheric muons as test particles. The measured rejection efficiency was about $95 \%$ at muons peak ( $\sim 55 \mathrm{MeV})$ (Ryumin et al., 1996). For low energies, the efficiency of the active veto is higher, since at these energies charged particles produce a smaller amount of light in the CsI(Tl) and the anticoincidence plastic scintillator makes a passive barrier for electrons with energies $<4 \mathrm{MeV}$ and protons with energies $<48 \mathrm{MeV}$.

The scintillation crystal had no collimator and its axis was parallel to the longitudinal axis of the satellite which was directed towards the Sun. Due to attenuation in the instrumental and spacecraft matter around the main detector, the nominal omnidirectional response for incoming X-rays would be reduced. According to the approach given in Bučík et al. (2002), an estimate of the detector field of view is $1.7 \pi \mathrm{sr}$ for $100 \mathrm{keV}$ and $2.3 \pi \mathrm{sr}$ for $1000 \mathrm{keV} \mathrm{X}$-ray energies. The calculation is based on a model of the distribution of matter near the CsI crystal in a similar experimental setup as for the preceding CORONAS-I experiment (Bučík and Kudela, 2003).

Energy losses in the scintillation crystal were pulseheight-discriminated into twelve differential channels from $30 \mathrm{keV}$ to $200 \mathrm{MeV}$, and one integral channel above $200 \mathrm{MeV}$. The SONG instrument provided high time resolution measurements of $1 \mathrm{~s}$ in the burst mode and $4 \mathrm{~s}$ in monitoring mode. For the present analysis we selected the four lowest energy channels (30-60, 60-150, 150-500, and $500-1500 \mathrm{keV})$. The instrument's maximum effective area (at vertical incidence) for gamma-ray photons is $270 \mathrm{~cm}^{2}$; the effective photopeak area at $100 \mathrm{keV}$ is $200 \mathrm{~cm}^{2}$, and $140 \mathrm{~cm}^{2}$ at $1 \mathrm{MeV}$. More details of SONG aboard CORONAS-I are given in Baláž et al. (1994). 

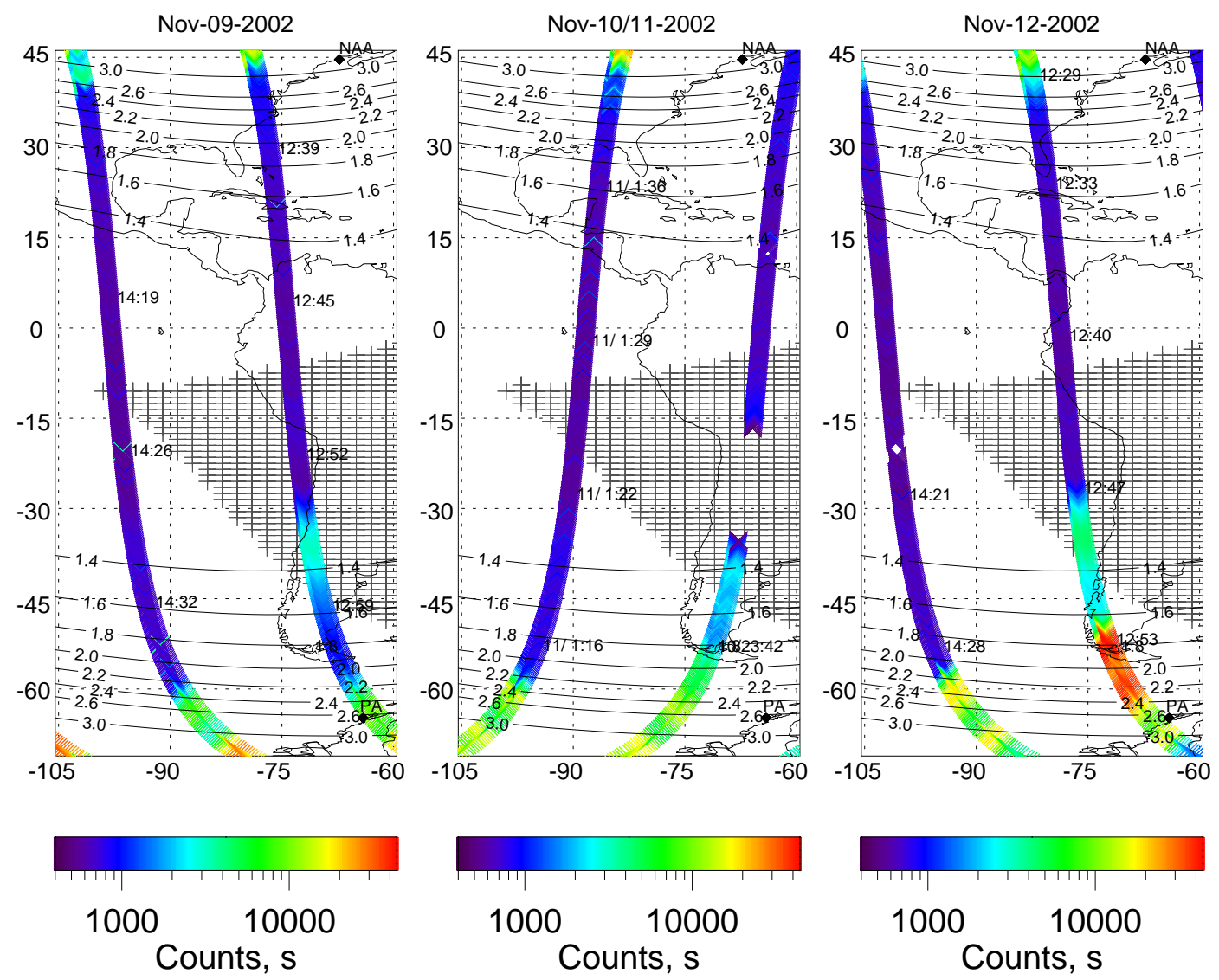

Fig. 2. Geographic locations of $X$-ray counts $(60-150 \mathrm{keV})$ during two consecutive CORONAS-F orbits displayed in each panel the times, shown in hours and minutes, UT. Footprints of several $L$ shells and stable trapped region (crosshatched) at $500 \mathrm{~km}$ are shown for reference, along with the location of the Palmer station (PA) and NAA VLF transmitter. Here we have data with the time resolution of $4 \mathrm{~s}$.

The SONG device was mounted on the platform at a distance of one meter from the satellite. Note that a fraction (as yet undetermined) of the detected hard X-rays was generated by interactions between primary cosmic rays and radiation belt particles with the CORONAS-F spacecraft and the SONG device itself.

\section{Observations}

An overview of hard X-rays in the $60-150 \mathrm{keV}$ energy channel, measured by CORONAS-F during October-December, 2002, is given in the map presented in Fig. 1. The data have been divided into $180 \times 180$ longitude-latitude bins with a width of $2^{\circ}$ in longitude and $1^{\circ}$ in latitude. The average number of measurements in one individual bin is $83 \pm 26$ and the total amount of the data is $2.5 \times 10^{6}$. Inspection of the map shows enhanced emissions in the slot region, $L \sim 2-3$, where, according to the predictions of Abel and Thorne (1998), scattering by lightning-generated whistlers dominates. One can see that in the closest vicinity of the South Atlantic Anomaly, between the longitudes of $-100^{\circ}$ and $-50^{\circ}$, the enhancements extend below $L \sim 2$.

One of the most intense enhancements, also seen in the inner zone, was found on 12 November 2002 (day 316) at around 12:55 UT ( $\sim 08: 00 \mathrm{LT})$ on the west side of the South Atlantic Anomaly in longitudes from $-75^{\circ}$ to $-65^{\circ}$. It is displayed in Fig. 2 (rightmost panel), where intense X-ray emissions (about 50 times the background value) in the energy channel of $60-150 \mathrm{keV}$ cover a rather wide $L$-shell range from 1.7 to 2.6. As shown in Fig. 3, the observed enhancement is characterized by a double-peak profile between 30 and $500 \mathrm{keV}$. The right panel of Fig. 2 shows that emissions at $L \sim 2-2.6$ gradually increase towards the South Atlantic Anomaly, and are similar to those in the atmospheric drift loss cone where the fluxes build up as electrons drift eastward due to injections over a range of longitudes (see, for example, Blake et al., 2001). In contrast, enhancements below $L \sim 2$ are probably localized in longitude; there we have no enhanced flux westward of longitude $-90^{\circ}$, although these were observed $1.5 \mathrm{~h}$ later, at 14:20 UT, on day 316 (see also Fig. 4, for other energy channels). 


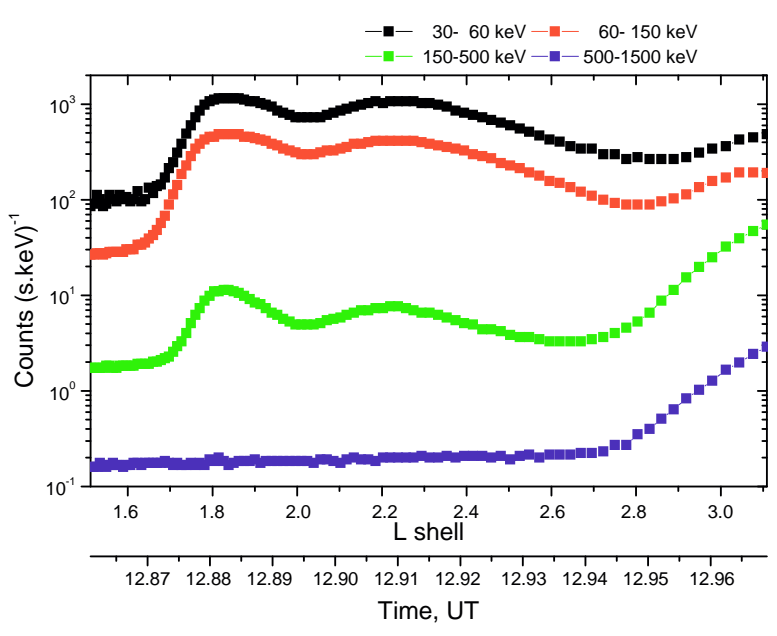

Fig. 3. X-ray counting rates versus $L$ shell/time in four energy channels measured by SONG during one passage (orbit on right, at longitudes between $-75^{\circ}$ and $-65^{\circ}$, rightmost panel of Fig. 2) of the CORONAS-F satellite on day 316 (12 November), the time being shown in hours, to two decimal places.

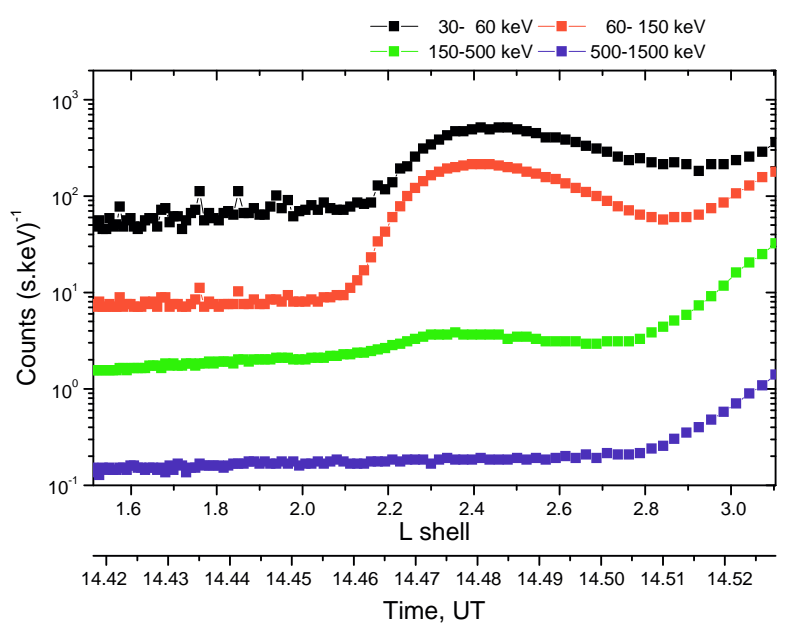

Fig. 4. Same as Fig. 3 for another passage (orbit on left, at longitudes between $-100^{\circ}$ and $-85^{\circ}$, right-hand panel of Fig. 2) on day 316 (12 November).

The $L$-shell profile of X-ray counts in Fig. 3 shows a relatively narrow peak in the inner zone (during the $\sim 90$-s satellite passage) centered on $L \sim 1.8$, and a broad maximum in the inner part of the slot region. Similar observations with a double-peak feature in connection with lightning-induced electron precipitation have been reported by Blake et al. (2001). They proposed that the narrow, low $L$ peak is attributed to resonant scattering at the first equatorial crossing at low $L$ shells, due to focusing of lightninggenerated whistler waves by particular ionospheric electron density gradients; the high $L$-shell broad structure is caused by whistlers undergoing multiple magnetospheric reflections (also see Bortnik et al., 2002).

During October-December 2002 there were few other similar intense emissions for $L<2$ in the vicinity of South Atlantic Anomaly. However, for this particular event, we have found good conjunctions with the Tropical Rainfall Measuring Mission (TRMM) satellite for performing a comparison with optical observations. The TRMM documents lightning activity by a Lightning Imaging Sensor (LIS), which locates lightning within its $600 \times 600 \mathrm{~km}$ field of view, with a 2-ms timing resolution at an altitude of $350 \mathrm{~km}$ (Christian et al., 1999). TRMM data show that an extensive thunderstorm system on 12 November 2002 (Fig. 5, rightmost panel), located in the longitude range of $-90^{\circ}$ to $-70^{\circ}$ in the Northern Hemisphere, within the $L$-shell range of 1.8-2.4, was active at least within the 11:00-12:45 UT interval. We note that lightning discharges were seen there during successive crossings of this area, up to the middle of the next day. The presence of the conjugate positions of the lightning flashes westward of the X-ray peak location suggests precipitation into the drift loss cone, although localized in longitude. An illustration of the atmospheric $100-\mathrm{km}$ loss cones for $L=1.8$ drift shell (similar to Fig. 1 of Sheldon et al. (1987) for $L=4$ ) is shown in Fig. 6. Electrons precipitated from the drift loss cone to the atmosphere, at a longitude of $\sim-70^{\circ}$, must be scattered from stable trapping by discharges located somewhere west of this longitude. In the northern lightning region, the satellite was in the bounce loss cone, and no enhancements of the X-ray count rate were noted below $L \sim 3$.

Figure 2 (leftmost panel) demonstrates that there is no (significant) enhancement at the same $L$ shell and longitude range, three days before (on 9 November 2002, day 313, $\sim 13: 00 \mathrm{UT}$ ), in the energy range of $60-150 \mathrm{keV}$, or for the other energy channels (Fig. 7). On day 313, the TRMM satellite passed through the region of interest in the north around 12:15 UT without indicating any lightning activity (Fig. 5, left panel).

The SONG instrument also observed enhanced X-ray counts on 10 November 2002, at around 23:45 UT, as shown in the middle panel of Fig. 2. The closest time when TRMM passed through the area of interest was on 10 November (blue curve in middle panel of Fig. 5) at 16:10 UT, more than $7 \mathrm{~h}$ earlier, without observing any significant lightning activity. However, on 11 November 2002 a large thunderstorm area (red crosses) had already built up between 11:55 and 12:03 UT, covering the longitudes between $-92^{\circ}$ and $-73^{\circ}$, as was shown by the LIS instrument.

\section{Internal detector background}

In the regions of the high fluxes of charged particles which can lose their energy in the gamma-ray detector, the measurement of X/gamma-ray fluxes, several orders lower than the charged particle fluxes, becomes quite difficult. In this 

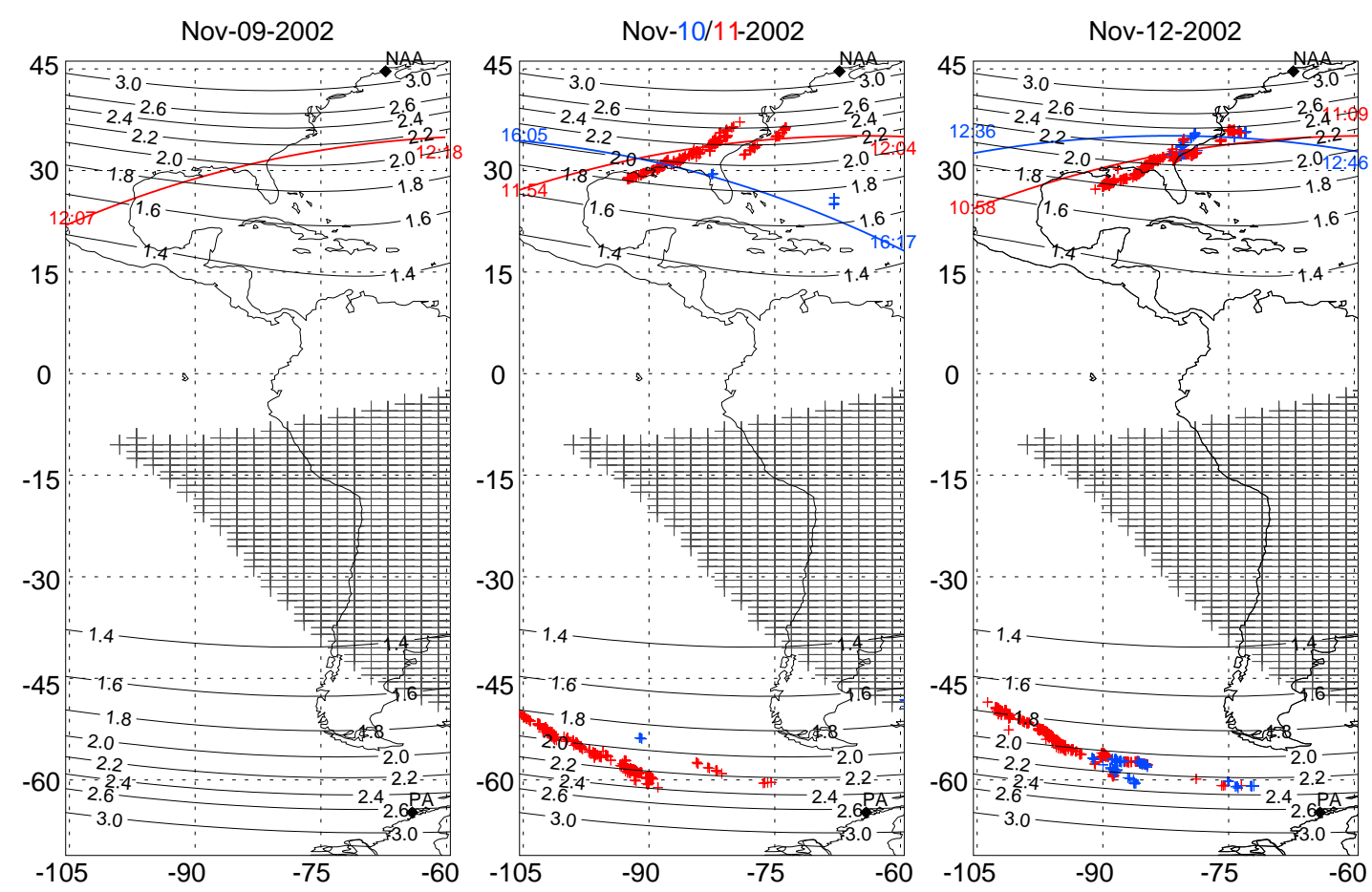

Fig. 5. Lightning discharges detected by the LIS aboard TRMM are shown as a red/blue crosses, together with respective TRMM trajectories indicated by solid red/blue curves. The crosses in the south indicate magnetically conjugate points (using IGRF 2000 geomagnetic field model) of the northern lightning flashes. Note that, for $L \sim 1.8$, the $150 \mathrm{keV}$ electrons need $\sim 20$ min to drift from a longitude of conjugate flashes at $-100^{\circ}$ to their loss from radiation belts at $-70^{\circ}$.

section we briefly discuss whether SONG observations in the X-ray energy channels between 30 and $500 \mathrm{keV}$ are due to photons and/or false signals caused by unvetoed charged particles.

For production of the bremsstrahlung X-ray photons with energies between $30 \mathrm{keV}$ and $500 \mathrm{keV}$, electrons with energies between $60 \mathrm{keV}$ and $1000 \mathrm{keV}$ are needed (Schlickeiser, 1981). The maximal flux of the $60-1000 \mathrm{keV}$ model AE8 electrons, mirroring at an altitude of $500 \mathrm{~km}$ in the inner belt, is about $\sim 10^{6} \mathrm{~cm}^{-2} \mathrm{~s}^{-1}$ (Vette, 1991). The flux of 30$500 \mathrm{keV}$ hard X-rays produced in the atmosphere by these electrons is about $10^{3}$ times lower, as provided by EGS4 simulations (Blake et al., 2002). Therefore, an efficient veto system for rejection of the high fluxes of charged particles is required.

The 2-cm thick plastic scintillator used in the SONG instrument would be opaque for the penetration of these electrons into the CsI crystal. For example, an average path length (which is less than penetration depth) for $500 \mathrm{keV}$ electrons in the plastic scintillator presents about $10 \%$ (Berger and Seltzer, 1982) of the SONG veto thickness. However, an electron having energy above $4 \mathrm{MeV}$, capable of penetrating the SONG plastic shield, could lose a fraction of its energy in the CsI and cause a false signal in the range of $30-500 \mathrm{keV}$. This contribution to the internal back- ground can be considered negligible. According to AE-8, the maximal integral flux of $>4 \mathrm{MeV}$ electrons at $90^{\circ}$ pitch angles at an altitude of $500 \mathrm{~km}$ in the inner belt is less than $1 \mathrm{~cm}^{-2} \mathrm{~s}^{-1}$. This value presents only a small fraction of the bremsstrahlung photon flux produced by $<1 \mathrm{MeV}$ inner belt electrons.

Since, for lower electron energies, bremsstrahlung generation of hard X-rays is a rather inefficient mechanism (considering the factor $10^{-3}$ ), the efficiency of the active veto should be greater than $99.99 \%$, in order to have an internal background which is quite insignificant (less than 10\%). Not knowing the exact value of the SONG veto efficiency in the low energy domain, we must admit that, observed outside the stable trapping region, enhanced counting rates between 30 $500 \mathrm{keV}$ may contain contributions from both electrons and hard X-rays. Further work is needed to specify the contribution of electrons to the internal background for the SONG detector.

\section{Discussion}

The lightning flashes on 11-12 November 2002, shown in Fig. 5, were most likely associated with the Major Severe Weather Outbreak in the Eastern USA, one of the largest severe weather events of the past 25 years. This occurred 


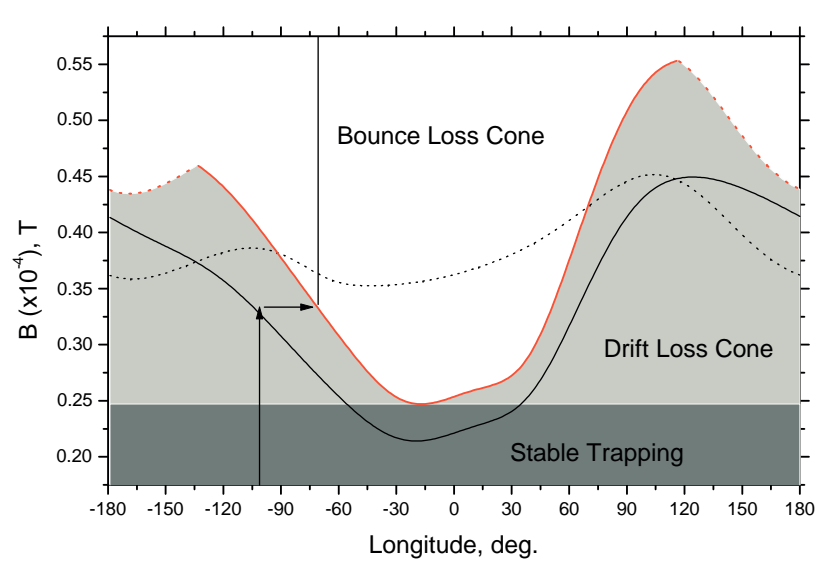

Fig. 6. Illustration of the $100-\mathrm{km}$ atmospheric loss cones for $L=1.8$. Electrons scattered into the drift loss cone are lost into the atmosphere in the Southern Hemisphere at longitudes between $-135^{\circ}$ and $-20^{\circ}$ (solid red curve), and in Northern Hemisphere between $120^{\circ}$ and $\sim 180^{\circ}$ (dotted red curve). Approximate satellite locations at $500 \mathrm{~km}$ in the south (north) are indicated solid (dotted) black curve. The schematic drawing shows that drift loss cone precipitation in the Northern Hemisphere requires a major change in magnetic field strength (i.e. in pitch angle) to transport electrons from stably trapped orbits. Vertical arrow at the longitude of $-100^{\circ}$, the location of the conjugate flashes for the event on 12 November schematically represents scattering from the stable trapping to the drift loss cone. The direction of the electron drift is marked by a horizontal arrow, and a vertical line at $-70^{\circ}$ indicates the longitude of the observed hard X-ray enhancements.

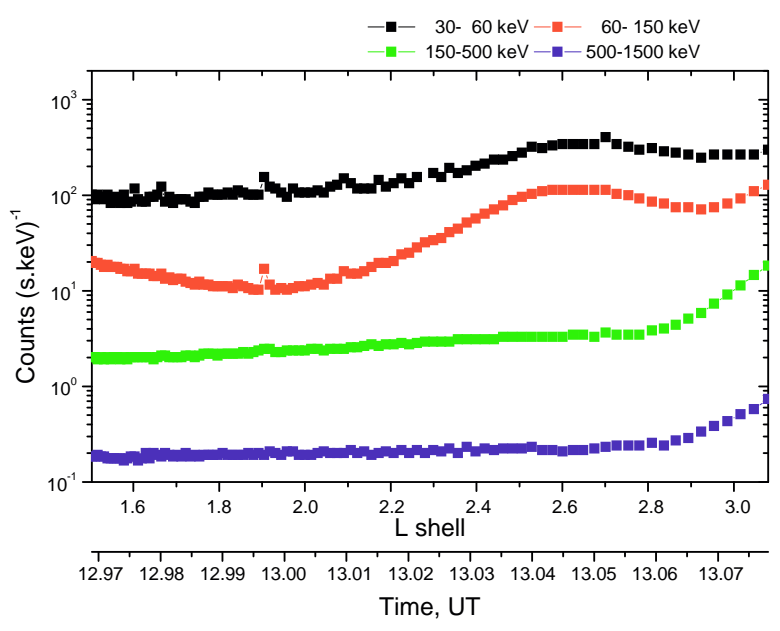

Fig. 7. Same as Fig. 3 for one passage on day 313 (9 November).

across much of the eastern United States on 10-11 November 2002, and was accompanied by many tornadoes and thunderstorms, as well as damage, and resulted in 36 deaths in 13 states (http://lwf.ncdc.noaa.gov). Infrared GOES images (http://cdo.ncdc.noaa.gov) on 12 November 2002 at
00:15 UT document the cloud covers over the location of the lightning strokes (not shown).

Before the start of the reported severe weather event, the $\mathrm{X}$-ray flux enhancement was observed only in the outer part of the slot region $(L \sim 2.5-3)$. After the appearance of thunderstorm clouds and associated lightning, demonstrated by GOES and TRMM, new X-ray emissions arose close to the magnetic conjugates of lightning under the inner belt region and in the inner part of the slot zone. These X-ray enhanced emissions were detected in three energy channels over 30 and $500 \mathrm{keV}$. We suppose that whistler waves launched by localized lightning in the north propagate to the southern conjugate region and are in cyclotron resonance with energetic trapped electrons which are moving northward. Due to the north-south asymmetry in mirror heights, this interaction lowers the mirror points into the Earth's atmosphere in the Southern Hemisphere (directly or, more likely, via azimuthal drift), where X-rays are produced through the bremsstrahlung process, which can emerge from the atmosphere to a low altitude orbit. Since $L$-dependent counting rates of the energetic electrons precipitating from the radiation belts have been attributed to the wave-particle interactions, the observed multiple-peak (double) structure over the $L$ shells may support our assumption.

From satellite observations, Datlowe and Imhof (1990) reported that most intense peaks of electron precipitation cluster at longitudes of major VLF transmitters. In the observed longitude range there is one high-power $(>110 \mathrm{~kW})$ communications transmitter currently in operation, namely, NAA (Maine, USA), located at a longitude of $-67^{\circ}$ and radiating at $24.0 \mathrm{kHz}$ with a power $\sim 1000 \mathrm{~kW}$. This transmitter signal could contribute, by resonance interactions with inner belt electrons, to the detected X-ray emissions. The calculations of Abel and Thorne (1998) indicate that 22.3-kHz VLF transmitter signals can resonate with $100-1500 \mathrm{keV}$ electrons below $L=2.2$. We note that VLF data from Palmer Station, Antarctica $(L \sim 2.6)$ documented activity of the NAA transmitter (http://www-star.stanford.edu/ $\sim$ palmer/) during times of the observed X-ray enhancements. A preliminary review of the data in October-December 2002, shows that in the majority of the passes through the longitudes around the NAA transmitter, no enhancements were seen under the inner radiation belt. It suggests that the contribution of NAA to the observations presented in this paper may be less important. Moreover, Cummer et at. (1997) supposed that, due to monochromatic VLF transmitter signals, the enhancements would be in a very narrow energy range. This is not the case in our observations, we detect enhanced counts over two orders of magnitude (tens to hundreds $\mathrm{keV}$ ).

Enhanced X-ray count rates, observed in the inner edge of the slot region may also suggest an injection of a new particle population. However, the mechanisms of the injection should be different from those operated during magnetic storms and substorms. No significant geomagnetic activity, as indicated by the $D s t$ index, was observed during and several days be- 
fore the event. The runaway avalanche acceleration (Gurevich et al., 1992), producing energetic electron beams in intense electric fields above the thunderstorms and their subsequent trapping by the geomagnetic field, can lead to the precipitation in the hemisphere conjugate to lightning (Lehtinen et al., 2001). Feldman et al. (1996) speculated that hard $\mathrm{X}$-ray enhancements observed near the geomagnetic equator, where there is not a significant population of permanently trapped electrons, could be due to newly-injected electrons into the drift loss cone of the inner radiation belt by upward lightning.

The detected increases on 12 November 2002 at $\sim$ 13:00 UT, when CORONAS-F was near local dawn ( 08:00 LT), are probably not associated with solar X-ray flares. GOES data do not report any class type of X-ray flare (Coffey, 2002) around the time of detection of the Xray counts peaks.

\section{Summary}

In this paper, we studied one intense enhancement of counting rates in energy channels measuring X-rays from 30 to $500 \mathrm{keV}$ for which observations of lightning flashes from space were available. We have found that:

- The spatial coincidence of the location of the lightning flashes with conjugate X-ray enhancements, and their simultaneity, suggests that CORONAS-F observed Xray counts are most likely connected to the underlying long-lived lightning documented by TRMM optical observations.

- Both the location of conjugate lightning flashes at longitudes west of the enhancements and the counts increasing toward the east are consistent with the expectations of the precipitation of electrons, which gradient drift eastward in longitude.

- The observed $L$-shell profile suggests that the enhancements may result from wave scattered energetic electrons, precipitated into the Earth's atmosphere, which, in turn, may generate bremsstrahlung X-rays.

Although we have observed an event which is definitely associated with lightning, further work is required to establish its statistical significance. We are currently investigating SONG/CORONAS-F data from the entire mission ( $\mathrm{Au}-$ gust 2001-November 2005) and are looking for conjunctions with TRMM satellite during this period. Further work also requires us to discuss simultaneous measurements of the wave environment.
Acknowledgements. This work was supported by the Slovak Research and Development Agency under the contract No. APVV51-053805. R. Bučík wishes to acknowledge VEGA grant 2/4064. We thank B. Yu. Yushkov and V. G. Kurt at INP MSU for the processing the SONG instrument data. The locations of the lightning flashes were obtained from the NASA Web site http://thunder.nsstc. nasa.gov/.

\section{References}

Abel B. and Thorne, R. M.: Electron scattering loss in Earth's inner magnetosphere 1. Dominant physical processes, J. Geophys. Res, 103, 2385-2396, 1998.

Baláž, J., Dmitriev, A. V., Kovalevskaya, M. A., Kudela, K., Kuznetsov, S. N., Myagkova, I. N., Nagornikh, Yu. I., Rojko, J., and Ryumin, S. P.: Solar flare energetic neutral emission measurements in the project CORONAS-I, IAU Colloq. 144, Solar Coronal Structures, edited by V. Rušin, P. Heinzel, and J.-C. Vial, VEDA, Bratislava, 635-639, 1994.

Berger, M. J. and Seltzer, S. M.: Stopping powers and ranges of electrons and positrons, National Bureau of Standards (2nd Ed.), Report NBSIR 82-2550-A, 1982.

Blake, J. B., Inan, U. S., Walt, M., Bell, T. F., Bortnik, J., Chenette, D. L., and Christian, H. J.: Lightning-induced energetic electron flux enhancements in the drift loss cone, J. Geophys. Res., 106, 29733-29 744, 2001.

Blake, J. B., Looper, M. D., Lorentzen, K. R., Kudela, K., and Bučík, R.: Correlation of spacecraft observations of energetic gamma ray fluxes with those of relativistic electrons in the drift loss cone, Geophys. Res. Abs., 4, 2002.

Bortnik, J., Inan, U. S., and Bell, T. F.: $L$ dependence of energetic electron precipitation driven by magnetospherically reflecting whistler waves, J. Geophys. Res., 107, 1150, doi:10.1029/2001JA000303, 2002.

Bučík, R., Kudela, K., Dmitriev, A. V., Kuznetsov, S. N., Myagkova, I. N., and Ryumin, S. P.: Spatial distribution of low energy gamma-rays associated with trapped particles, Adv. Space Res., 30, 2843-2848, 2002.

Bučík, R. and Kudela, K.: On mass in $4 \pi$ solid angle around SONG CsI scintillator aboard CORONAS-I satellite, Acta Phys. Slov., 53, 329-345, 2003.

Bučík, R., Kudela, K., Kuznetsov, S. N., and Myagkova, I. N.: Gamma rays in $L-B$ coordinates at CORONAS-I altitude, Ann. Geophys., 23, 2239-2247, 2005.

Christian H. J., Blakeslee, R. J., Goodman, S. J., et al.: The Lightning Imaging Sensor, in: Proceedings of the 11th International Conference on Atmospheric Electricity, Guntersville, Alabama, June 7-11, 746-749, 1999.

Coffey, H. E. (Ed.): Solar-Geophysical Data, 200211, Part II, (http: //sgd.ngdc.noaa.gov/sgd/jsp/solarindex.jsp), 2002.

Cummer, S. A., Bell, T. F., Inan, U. S., and Chenette, D. L.: VLF remote sensing of high-energy auroral particle precipitation, J. Geophys. Res., 102, 7477-7484, 1997.

Datlowe, D. W., and Imhof, W. L.: Cyclotron resonance precipitation of energetic electrons from the inner magnetosphere, J. Geophys. Res., 95, 6477-6491, 1990.

Feldman, W. C., Symbalisty, E. M. D., and Roussel-Dupré, R. A.: Hard X ray survey of energetic electrons from low-Earth orbit, J. Geophys. Res., 92, 2505-2513, 1996. 
Foat, J. E., Lin, R. P, Smith, D. M., et al.: First detection of a terrestrial MeV X-ray burst, Geophys. Res. Lett., 25, 4109-4112, 1998.

Goldberg, R. A., Curtis, S. A., and Barcus, J. R.: Detailed spectral structure of magnetospheric electron bursts precipitated by lightning, J. Geophys. Res., 92, 2505-2513, 1987.

Gurevich, A. V., Milikh, G. M., and Roussel-Dupré, R. A.: Runaway electron mechanism of air breakdown and preconditioning during a thunderstorm, Phys. Lett. A, 165, 463-468, 1992.

Kuznetsov, S. N., Kudela, K., Ryumin, S. P., and Gotselyuk, Y. V.: CORONAS-F satellite: tasks for study of particle acceleration, Adv. Space Res., 30, 1857-1863, 2002.

Kuznetsov, S. N., Kudela, K., Myagkova, I. N., Podorolsky, A. N., Ryumin, S. P., and Yushkov, B. Yu.: First experience with SONG-M measurements on board CORONAS-F satellite, Indian J. Radio Space Phys., 33, 353-357, 2004.

Lehtinen, N. G., Inan, U. S., and Bell, T. F.: Effects of thunderstorm-driven runaway electrons in the conjugate hemisphere: Purple sprites, ionization enhancements, and gamma rays, J. Geophys. Res., 106, 28 841-28 856, 2001.

Lorentzen, K. R., McCarthy, M. P., Parks, G. K., et al.: Precipitation of relativistic electrons by interaction with electromagnetic ion cyclotron waves, J. Geophys. Res., 105, 5381-5389, 2000.

Martin, I. M., Rai, D. B., Palmeira, R. A. R., Trivedi, N. B., Abdu, M. A., and da Costa, J. M.: Enhanced low-energy gamma rays at balloon altitude in the Brazilian magnetic anomaly, Nature, 252, 25-27, 1974.
Millan, R. M., Lin, R. P., Smith, D. M., Lorentzen, K. R., and McCarthy, M. P.: X-ray observations of $\mathrm{MeV}$ electron precipitation with a balloon-borne germanium spectrometer, Geophys. Res. Lett., 29, 2194, doi:10.1029/2002GL015922, 2002.

Rosenberg, T. J., Helliwell, R. A., and Katsufrakis, J. P.: Electron precipitation with discrete very-low-frequency emissions, J. Geophys. Res., 76, 8448-8451, 1971.

Ryumin, S. P., Bogomolov, A. V., Dmitriev A. V., and Myagkova, I. N.: Background conditions for recording $\gamma$ radiation and energetic neutrons on CORONAS satellites from SONG data, J. Moscow Phys. Soc., 6, 423-430, 1996.

Schlickeiser, R.: Gamma ray astronomy and the origin of cosmic rays, Fortschritte der Physik, 29, 95-134, 1981.

Sheldon, W. R., Benbrook, J. R., Bering, E. A., Leverenz, H., Roeder, J. L.: Electron precipitation near $L=4$ - Longitudinal variation, Adv. Space Res., 7, 49-52, 1987.

Smith, D. M., Lin, R. P., Anderson, K. A., Hurley, K., and Johns, C. M.: High-resolution spectra of $20-300 \mathrm{keV}$ hard X-rays from electron precipitation over Antarctica, J. Geophys. Res., 100, 19675-19685, 1995.

Vette, J. I.: The AE-8 trapped electron model environment, NSSDC 91-24, NASA Goddard Space Flight Center, Greenbelt, Md., 1991.

Voss, H. D., Walt, M., Imhof, W. L., Mobilia, J., and Inan, U. S.: Satellite observations of lightning-induced electron precipitation, J. Geophys. Res., 103, 11 725-11 744, 1998. 